Thorax, 1981, 36, 99-107

\title{
Auscultation of the lung: past lessons, future possibilities
}

\author{
RAYMOND L MURPHY \\ From the Pulmonary Service, Lemuel Shattuck and Faulkner Hospitals, \\ Boston, Massachusetts, USA
}

\begin{abstract}
Review of the history of auscultation of the lung reveals few scientific investigations. The majority of these have led to inconclusive results. The mechanism of production of normal breath sounds remains uncertain. Hypotheses for the generation of adventitious sounds are unproven. Advances in instrumentation for lung sound recording and analysis have provided little of clinical value. There has been a recent resurgence of interest in lung sounds. Space-age technology has improved methodology for sonic analysis significantly. Lung sounds are complex signals that probably reflect regional lung pathophysiology. If they were understood more clearly important non-invasive diagnostic tools could be devised and the value of clinical auscultation could be improved. A multidisciplinary effort will be required to achieve this.
\end{abstract}

\begin{abstract}
"Every adversity has within it the seed of an equivalent or even greater benefit." (N Hill)

Laënnec revolutionised the diagnosis of lung disease with an instrument that is imprecise by today's standards. Clues to the explanation for this surprising finding can be found in the following review of lung sound literature. Although much has been written on lung sounds, few scientific investigations have been reported. In this article I will focus on studies of the mechanism, production, and transmission of normal and abnormal lung sounds as well as methods for objective analysis of these sounds. These scientific studies provide interesting lessons and inspire speculation on the potential value of a more complete understanding of pulmonary auscultation.
\end{abstract}

\section{Origin of vesicular and bronchial sounds}

Laënnec ${ }^{1}$ described the normal lung sound as "a slight but extremely distinct murmur answering to the entrance of the air into and its expulsion from the air cells of the lungs." Early speculation included the following: (1) the noise was made all along the respiratory tract by friction of gases against the airway walls; (2) the glottis alone caused the noisethe sound heard over the chest wall was the glottic sound attenuated during transmission through the

Address for reprint requests: Dr RLH Murphy, Director, Pulmonary Service, Faulkner Hospital, 1153 Centre Street, Boston, MA 02130 , USA. lung; (3) the sound was made as air passes from a narrower to a wider space on respiration. Bullar ${ }^{2}$ tested these hypotheses by using an artificial thorax consisting of an air-tight, fluid-filled chamber with glass sides. He could expand and contract this chamber at will with a bellows. He placed one of two sheep lungs connected to each other by the trachea and bronchi into the artificial thorax, the other being left outside. With the trachea open, operation of the bellows ventilated the lung in the box while the outer lung lay still and collapsed. Vesicular sounds were heard over the lung in the box; bronchial sounds were noted over the outer lung. The well-recognised phenomenon of sound production when an air current passes over but not into the mouth of a tube was considered the mechanism for the bronchial breathing in the outer lung. The trachea was then plugged so that during ventilation of the inner lung the air was displaced in and out of the outer lung. Vesicular sounds were then heard over the outer lung indicating that they were not produced in the glottis or trachea alone. Bullar also demonstrated that no sound was generated despite fluctuations in lung volume and pressure under conditions of no flow. He concluded that sounds are produced at those parts of the respiratory tract where air passes from a narrower to a wider space. Thus, during inspiration one sound is produced at the glottis and another at the points where the bronchioles open into "the vesicles." 
Hannon et $a l^{3}$ investigated the site of lung sound production by placing exenterated sheep lung over the neck of a human and recording the frequency characteristics of the sounds heard over the sheep lung and over the back in the tenth intercostal space. They found no vibrations below $130 \mathrm{~Hz}$ over the sheep's lung but such vibrations were simultaneously detectable at the tenth intercostal space. They concluded that ventilation of lung underlying the stethoscope was necessary to produce this low frequency sound

As sound filtering techniques were developed they began to be applied to lung sound analysis. German investigators tried to relate the frequent characteristics of bronchial breathing to the size of the airway in which they were produced. ${ }^{4}$ The underlying assumption was that the various portions of the tracheobronchial tree vibrate on excitation at fundamental frequencies related to their dimensions. They believed that such knowledge would provide information on the location and extent of consolidated lung tissue. Bronchi or the model rubber tubes they studied, which had internal diameters of $3 \mathrm{~mm}$ or less, were poor conductors of sound of the frequency of bronchial breath sounds. Since they were unable to record vibrations of $\mathbf{1 7 0 0}$ or greater in bronchial breath sounds they concluded that airways $3 \mathrm{~mm}$ in diameter or less take no part in the formation of such sounds. They concluded that consolidated lung tissue had to extend from the pleural surface to the conducting airways of $2-3 \mathrm{~mm}$ in size to hear the bronchial sounds. Their results correlated with measurements in cadaver lungs and clinical findings in pneumonia.

Increased information on pulmonary function, especially the mechanics of breathing, led to attempts to use this knowledge in interpreting lung sound production. Forgacs ${ }^{5}$ believed that the source of the lung sounds is the turbulence in the airstream flowing through the pharynx, glottis, and large airways. The extent of the turbulence peripherally depends on the flow rate, but even in the resting subject there is often turbulence in the main bronchi. He called attention to the clinical observation that breath sounds in some subjects are silenced synchronously with the heartbeat while they are heard continuously on the opposite side. He deduced from this that part of the noise must be generated at least as far peripherally as the main bronchi and possibly in more peripheral branches as well. This component of the breath sounds is suppressed when the velocity of the airstream entering the left lung is momentarily slowed by air driven in the opposite direction by pulsation of the heart. Forgacs ${ }^{6}$ quantified the increase in sound at the mouth in bronchitis and asthma and the absence of such an increase in primary emphysema. This also argued for an infra- $\frac{2}{2}$ glottic source of the inspiratory sound especially as $\frac{C}{0}$ the increased intensity of the respiratory sound was $\overline{\bar{D}}$ also present over the stoma of a patient with bron- $\widehat{\Phi}$ chitis who had undergone laryngectomy. When a helium mixture was breathed by these patients the ${ }^{\infty}$ inspiratory sound became quieter, consistent with $\vec{O}$ the theory that turbulence was the cause of the sound. $\vec{\overrightarrow{ }}$ Forgacs argued that calculations from the rate of $\vec{\sigma}$ volume flow at the mouth and the total cross-section $\overrightarrow{\overrightarrow{2}}$ of the airways at each generation of the bronchi $\vec{\omega}$ indicated that the flow velocity beyond the lobar $\stackrel{\omega}{\circ}$ or segmental bronchi is too low to generate turbulence. In the more peripheral branches flow 6 was laminar and presumably silent.

Hardin and Patterson ${ }^{7}$ applied flow visualisation $\rightarrow$ techniques to the study of the behaviour of air in a $\mathbb{D}$ model of a typical junction in the human bronchial 을 tree. Vortices were formed at each junction at Reynold's numbers in the range $50-4500$ corresponding to those found in the fifth through thirteenth $\vec{\oplus}$ generations of the bronchial tree. Combining this $\stackrel{\infty}{\rightarrow}$ with the theory of vortex sound generation, they 0 concluded that the frequencies of sound produced by these vortices corresponded to those seen on spectral analysis of lung sounds. Furthermore, they stated that the frequency of sound produced at each $\mathbb{D}$ vortex was related to the flow velocity and diameter of the tube. Thus, they believed that the order of bronchi in which particular frequencies are generated can be determined. Unfortunately, the proof that the vortices themselves produce the sound or that the spectral components of the lung sounds they analysed came from the size bronchi they claim is not convincing.

To study the site of production of breath sounds, Kraman ${ }^{8}$ made simultaneous recordings from two different areas of the chest. These were then analysed by subtraction pneumography to determine whether they were similar sounds, and therefore, transmitted from a distant source or dissimilar, and therefore, transmitted from sources located near each microphone. His findings using microphones separated o by $10 \mathrm{~cm}$ were consistent with an intrapulmonary $N$ source for the inspiratory component and an upper $N$ airway source for at least some of the expiratory component. This work represents a clever application of a standard acoustic technique to help identify the site of lung sound production.

Curiously, investigators who actually performed experiments to observe sounds concluded that some sound is generated peripherally despite the theoretical arguments that suggest that air movement in the periphery is silent. Were the answer known it $\Omega$ would allow a more precise estimate of how useful lung sounds are in estimating regional ventilation. 
A discussion of this topic, however, requires consideration of the sound conducting properties of the lung.

\section{Transmission of sounds in the chest}

Bullar $^{2}$ had observed in his model that bronchial sounds were attenuated when the inner lung was distended and louder when it was collapsed, consistent with the clinical observation that bronchial sounds are attenuated by air filled lung. The graphic representations of respiratory sounds of Hannon and Lyman $^{3}$ were made (1) directly over the trachea, (2) transmitted through a constant thickness of sheep's lung placed over the neck of the same subject, and (3) at the right base of the chest of the same subject. Interestingly, the sound they observed directly over the trachea in expiration without filters was louder than the inspiratory sound but the inspiratory sound below $130 \mathrm{~Hz}$ was louder than the expiratory sound below $130 \mathrm{~Hz}$.

Using a low pass filter to exclude vibrations greater than $130 \mathrm{~Hz}$ they observed low frequency vibrations over the trachea in a normal human. These were also present when an uninflated sheep's lung was interposed between the recording electrical stethoscope and the larynx of this subject. When the lung was inflated to a thickness of $4 \mathrm{~cm}$ these vibrations were not recorded no matter what the pressure in the lung. The low frequencies were visible on simultaneous recordings made at the right lung base. They concluded that the passage of air into and out of the lung was necessary for the transmission of these low frequency vibrations. Nairn and TurnerWarwick $^{9}$ in 1968 compared breath sound intensity to regional ventilation estimated by radioactive xenon. When sounds were poor or absent in emphysema there was almost invariably poor intrapulmonary mixing in the underlying lung zone. This is consistent with an intrapulmonary origin of the sound but does not exclude a central origin with regional alterations in transmission characteristics. LeBlanc, Macklem, and Ross ${ }^{10}$ studied breath sound intensity under controlled lung volumes, flows, and body positions. In subjects in the upright and lateral decubitus positions they found that maximal relative intensity of breath sounds varied in the same way as expected from studies of the regional distribution of ventilation observed with radioactive xenon. They believed that breath sound intensity was a good physical sign of regional pulmonary ventilation and presented evidence that the vesicular sound was related to alveolar filling. In contrast, Pioy-SongSang et al ${ }^{11}$ found poor correlation with a phonopneumographic breath sound index and regional distribution of xenon and concluded the breath sound heard by clinicians cannot be used to assess regional ventilation. They corrected the breath sound index by dividing it by an index of sound transmission obtained by introducing white noise at the mouth. The breath sounds so "compensated" were believed to reflect not only regional ventilation but also the number of sound generators under the inicrophone.

Rice $^{12}$ pointed out that sound propagates along the airways, with relatively little attenuation, at free field sound speeds in the gas, but that sound speeds in the lung were reported to be unusually slow. This suggested that the low velocities were caused by a propagation along a path much longer than the straight line distance between the sound source and receiver. He measured velocities ranging from 20 to 70 metres per second across excised horse lung. This was less than the known speed of sound in air $(300 \mathrm{~m} / \mathrm{s})$ or solid tissue $(1500 \mathrm{~m} / \mathrm{s})$. Inflation of the lung with high or low density gas changed the velocity only slightly, demonstrating that circuitous routes of sound propagation through airways were unlikely. The sound velocities he observed approximated those calculated assuming that the parenchy$\mathrm{ma}$ is a uniform fluid and that compressibility and density are the average of the gas and tissue phases. This is the most enlightening work on the subject to date, but awaits confirmation in humans. The relevance of transmission of sound across the lung to sound generated within it should also be clarified.

The study of lung sound generation and transmission in disease states is even more difficult than in normals because of the occurrence of adventitious or "added" sounds. The understanding of these latter sounds is further complicated by the problem of lung sound nomeclature.

\section{Nomenclature and observer variability}

Laënnec said lung sounds were easier to distinguish than describe. The difficulties in description have led both to a plethora of terms for the same sound and to distinctly different sounds being called by a single name. The confusion caused by the translation of L'Auscultation Mediate into English was described by Robertson ${ }^{13}$ in an elegant, albeit whimsical review. Confusion in terms is probably an important factor in observer variability. Perceptual differences between observers may also exist and the sounds themselves vary from breath to breath. In contrast, heart sounds are relatively uniform from beat to beat, perhaps accounting for their greater diagnostic utility. There have been relatively few studies to quantify the magnitude of observer variability. Most of these have focused on reliability or observer agreement 
rather than accuracy. Most studies have shown high disagreement, ${ }^{14-18}$ a problem in clinical data collection in general, ${ }^{18}$ including the interpretation of chest radiographs. ${ }^{19} 20$ Some studies $^{9} 21$ have shown fairly good agreement, perhaps by employing more rigid criteria and methodology or by focusing on single observations at a time. The accuracy of lung sounds in detecting obstructive lung disease appears to be better than other physical signs. ${ }^{21}{ }^{22}$ Accordingly, objective methods to quantify lung sounds have been sought.

\section{Lung sound recording and analysis}

Numerous attempts have been made to display sound visually. They have focussed on the amplitude of the sound over time, or temporal properties, and on analysis of the sound by component frequencies, or spectral properties. ${ }^{23} 35$ In 1924 Bass $^{36}$ reported a clever method using a condenser microphone and oscilloscope that displayed normal and abnormal lung sounds. Cabot and Dodge ${ }^{31}$ were able to show in 1925 that coarse and fine rales were associated with conspicuous low and high frequency components respectively. In 1955, McKusick et al ${ }^{23}$ demonstrated that the spectrograms of "moist "and "dry "crackling rales were different, the dry rales containing more high frequencies. Calibrated amplitude plots reported by Weiss and Carlson ${ }^{24}$ in 1970 allowed visual presentation of the overall amplitude of sounds, including pauses and duration differences as well as a study of the relation of the amplitudes in inspiration and expiration. Adventitious sounds were not clearly distinguished from one another by these methods nor by the integrating envelope detector described by Wooten and Waring. ${ }^{27}$

Speculations on the difficulty of visually differentiating lung sounds were presented by Forgacs in $1967 .{ }^{37}$ He pointed out that the study of crackling was difficult because the sounds follow one another so rapidly that neither the individual crackles, nor the rhythmical pattern made by their sequence can be identified with the unaided ear. He noted that the oscilloscope showed the regular waveforms of a wheeze but only at time base speeds at which one saw such a small section of sound that it was very difficult to judge the timing of the wheezes in relation to the respiratory cycle.

Table Outline of classification of lung sounds.

\begin{tabular}{|c|c|c|c|c|c|c|}
\hline Acoustic characteristics & Waveform & $\begin{array}{l}\text { Recommended } \\
\text { ATS* } \\
\text { nomenclature }\end{array}$ & $\begin{array}{l}\text { Terms in } \\
\text { some } \\
\text { textbooks }\end{array}$ & $\begin{array}{l}\text { A British } \\
\text { usage }\end{array}$ & $\begin{array}{l}\text { Laënnec's } \\
\text { original term }\end{array}$ & Laënnec's model \\
\hline $\begin{array}{l}\text { Discontinuous, } \\
\text { interrupted explosive } \\
\text { sounds } \\
\text { Loud, low in pitch }\end{array}$ & & Coarse crackle & Coarse rale & Crackle & $\begin{array}{l}\text { Rale muquex } \\
\text { ou gargouille- } \\
\text { ment }\end{array}$ & $\begin{array}{l}\text { Escape of water } \\
\text { from a bottle held } \\
\text { with mouth } \\
\text { directly downward }\end{array}$ \\
\hline $\begin{array}{l}\text { Discontinuous, } \\
\text { interrupted explosive } \\
\text { sounds } \\
\text { Less loud than above } \\
\text { and of shorter } \\
\text { duration; higher in } \\
\text { pitch than coarse rales } \\
\text { or crackles }\end{array}$ & & Fine crackle & $\begin{array}{l}\text { Fine rale } \\
\text { crepitation }\end{array}$ & Crackle & $\begin{array}{l}\text { Rale humide } \\
\text { ou crepitation }\end{array}$ & $\begin{array}{l}\text { Crepitation of } \\
\text { salts in a heated } \\
\text { dish. Noise } \\
\text { emitted by } \\
\text { healthy lung } \\
\text { when compressed } \\
\text { in the hand }\end{array}$ \\
\hline $\begin{array}{l}\text { Continuous sounds } \\
\text { Longer than } 250 \mathrm{~ms} \text {, } \\
\text { high-pitched; dominant } \\
\text { frequency of } 400 \mathrm{~Hz} \text { or } \\
\text { more, a hissing sound }\end{array}$ & & Wheeze & $\begin{array}{l}\text { Sibilant } \\
\text { rhonchus }\end{array}$ & $\begin{array}{l}\text { High-pitched } \\
\text { wheeze }\end{array}$ & $\begin{array}{l}\text { Rale sibilant } \\
\text { sec ou } \\
\text { sifflement }\end{array}$ & $\begin{array}{l}\text { Prolonged } \\
\text { whisper of various } \\
\text { intonations; } \\
\text { chirping of birds; } \\
\text { sound emitted by } \\
\text { suddenly } \\
\text { separating } 2 \\
\text { portions of } \\
\text { smooth oiled } \\
\text { stone. The } \\
\text { motion of a } 6 \text { mall } \\
\text { valve }\end{array}$ \\
\hline $\begin{array}{l}\text { Continuous sounds } \\
\text { Longer than } 250 \mathrm{~ms} \\
\text { low pitched; dominant } \\
\text { frequency about } 200 \mathrm{~Hz} \\
\text { or less; a snoring sound }\end{array}$ & & Rhonchus & $\begin{array}{l}\text { Sonorous } \\
\text { rhonchus }\end{array}$ & $\begin{array}{l}\text { Low-pitched } \\
\text { wheeze }\end{array}$ & $\begin{array}{l}\text { Rale sec } \\
\text { senore ou } \\
\text { ronflement }\end{array}$ & $\begin{array}{l}\text { Snoring; bass } \\
\text { note of a musical } \\
\text { instrument; } \\
\text { cooing of a wood } \\
\text { pigeon }\end{array}$ \\
\hline
\end{tabular}

*American Thoracic Society

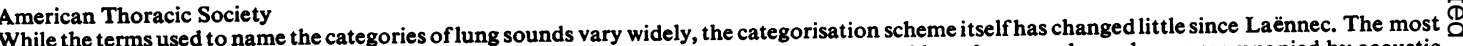
recent names recommended for adoption by the American Thoracic Society and terms used by others are shown here, accompanied by acoustic $\sigma$ descriptions and examples of typical sound waveforms for each category. 
Forgacs, ${ }^{37} 38$ and Nath and Capel ${ }^{39} 40$ described the repetitive nature of rales in some patients but their published tracings had time scales below $100 \mathrm{~mm} / \mathrm{s}$ and did not reveal clearly the waveform characteristics of the adventitious sounds. The amplitude deflections produced by rales, for example, are similar to deflections found in tracings in whch no rales are described since the specific waveforms of the rales are obscured at this speed.

Space-age technology has provided more sophisticated computer-based methods, such as time-

\section{A NORMAL TRACHEAL}

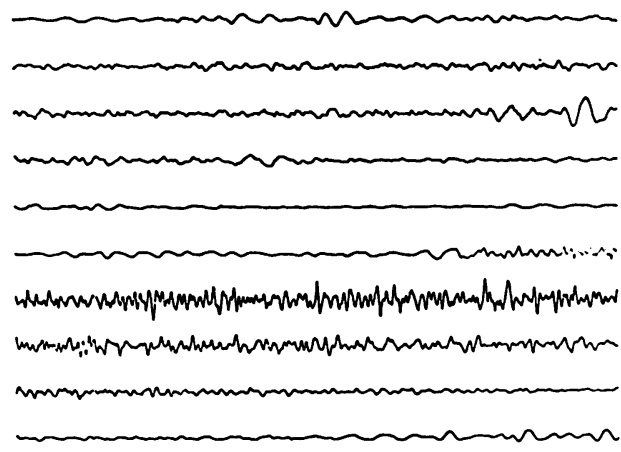

\section{B MORMAL VESICULAR}

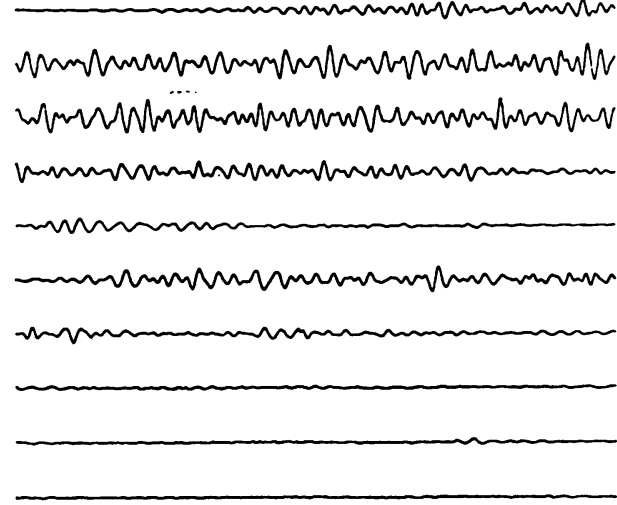

expanded waveform analysis, which have helped to solve some of the nomenclature problems (figure). Focusing on the objective description provided by authors as well as using time-expanded waveform analysis for verification has enabled the development of the classification outlined in the table. Laënnec, ${ }^{1}$ with his characteristic thoroughness, provided us with a variety of models. Time-expanded waveform analysis of sounds made by these models shows that they are similar to those of the lung sounds they were intended to represent. For example, sound produced

\section{C fine Crackles}

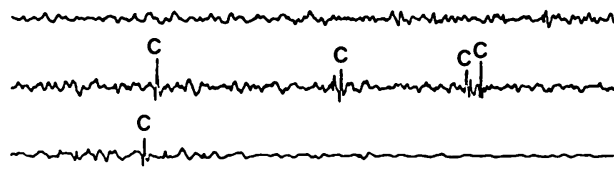

D WHEEZE

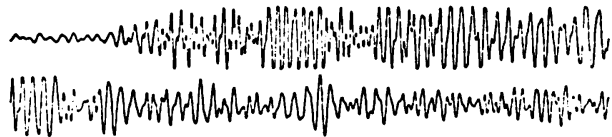

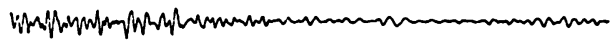

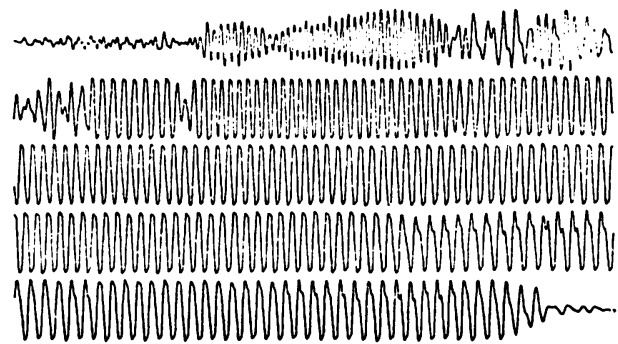

Figure Time-expanded waveforms of lung sounds

The time-intensity plots in this figure are made from tape recordings of lung sounds stored in a computer memory and then visually displayed on a two dimensional plot, with amplitude or intensity on the Y axis. The time axis of the plot is "expanded" or magnified by playing back slowly from the computer memory.Plots $A$ and $B$ illustrate normal tracheal and vesicular sounds. The louder, longer expiratory phase of the tracheal sound is readily recognised as is the pause between inspiration and expiration. When crackles occur, they produce intermittent ("discontinuous") deflections superimposed on the normal vesicular pattern (marked C in plot C). Plot shows a "continuous" deflection produced by a wheeze replacing the normal waveform. 
by heating salt in a frying pan shows intermittent spikes similar to those of fine crackles, while a musical note shows undulating sinusoidal deflections similar to a rhonchus. More rigorous analysis of lung sounds coupled with establishment of standards for the classical major categories should allow even further clarification of the nomenclature.

\section{DISCONTINUOUS SOUNDS}

Very few people have attempted to define either the sites or origin or the mechanism of production of added or adventitious sounds. The most clearly thought-out efforts in this regard are those of Forgacs. ${ }^{41}$ It seems likely that more than one generating mechanism for crackles exists. The coarse, discontinuous sound occurring in pulmonary oedema or other conditions in which fluid is found in the airways are probably caused by rupture of fluid films or bubbles. Forgacs ${ }^{37}$ has argued convincingly that crackles occurring in diseases such as pulmonary fibrosis or early congestive heart failure are not a result of air bubbling through fluid. His observations support instead the hypothesis that crackles in these diseases result from the sudden release of energy stored in the lung after delayed opening during inspiration of airways that had closed at the end of the previous expiration. He based his arguments on the often strikingly repetitive occurrence of crackles during succeeding inspirations, even after a cough. These crackles rarely occur in expiration and are often localised over dependent areas of the lung, where gravitational stress predisposes the airways to collapse at low lung volumes. Air bubbling through fluid would be expected to cause a noise during both inspiration and expiration. The pattern would probably change after a cough had redistributed the fluid.

\section{CONTINUOUS SOUNDS}

Wheezes are believed to be generated by a regular vibration, or oscillation, of the airway wall at one or more sites in the chest. Forgacs ${ }^{41}$ observed that the pitch of a wheeze is relatively independent of gas density. This indicates that wheezes are not produced in the same way as the note of an organ pipe, in which the pitch of the note depends both on the length of the pipe and on the density of the vibrating gas in the pipe. Forgacs also found that he could generate wheeze-like noises in excised bronchus by blowing air through the specimen and using compression to generate external pressure.

Thus, it seems likely that wheezes occur when air passing through a narrowed airway at high velocity produces a decrease in the gas pressure in the airway at the region of constriction. Bernoulli's principle relates the local velocity of the gas to local pressure in such flow. As the velocity of the gas increases at the constriction, the pressure decreases. If allowed by the other forces acting on the airway, collapse will continue progressively until there is a substantial resistance and the flow is decreased. Then the internal pressure increases and the lumen enlarges. This alteration of the wall of the airway between almost closed and almost open is believed to produce the wheezing noise.

Our understanding of adventitious sounds is even more incomplete than our knowledge about the normal sounds. Like the latter it is based on too few experiments and much speculation. The numerous studies that have attempted clinical correlation of lung sounds with disease states have been deficient. Most have had too few subjects, outdated equipment, or more importantly, conclusions that were imprecise. It is not surprising that an important text of pulmonary disease had the following comment: "The stethoscope is largely a decorative instrument in so far as its value in diagnosis of pulmonary diseases is concerned. Nevertheless, it occupies an important place in the art of medicine. Apprehensive patients with functional complaints are often relieved as soon as they feel the chest piece on their pectoral muscles." 42

\section{Current clinical uses}

It seems paradoxical that despite our incomplete information on lung auscultation and the availability of more accurate diagnostic tools, physicians continue to use the stethoscope routinely to examine the lungs. It is useful to consider the reasons for this. Lung sounds can be quite important in the daily bedside and outpatient management of patients with a variety of illnesses such as bronchial asthma and congestive heart failure. These sounds can provide crucial information in instances of upper airway obstruction, misplaced endotracheal tubes, and spontaneous pneumothorax. In contrast to many diagnostic techniques, auscultation is readily performed in a variety of settings. It is often used to help decide on the necessity for additional clinical studies. It is my belief that more precise knowledge would improve its accuracy in such instances. More importantly, understanding the nature of lung sounds could lead to improved non-invasive pulmonary diagnosis. The reasons for this belief will be considered now.

Areas for further investigation including potential future applications

An extremely complex sonic signal is sent by the lung during breathing. Modern analytic techniques provide the equivalent of an acoustic microscope. 
This allows study of the lung's sonic signal with a precision not previously available. This signal can be seen to vary with recording site, flow rate, lung volume, body position, and various breathing manoeuvres. It is likely that the sound changes with growth, development, and age, as well as with minimal environmental insults. The signal is so complex and varies so much that it appears at times to be random or unpredictable. It is more likely, however, that the sonic signal reflects the underlying anatomy and pathophysiology. Were it better understood, powerful diagnostic tools using lung sound maps might be developed. In contrast to pulmonary function studies, the information obtained is likely to be determined by regional pathophysiology. Lung sounds promise to reflect certain pathological processes such as the obstructive diseases and early interstitial processes more closely than radiological studies do. The credibility of such speculation is enhanced by considering the advances that came in chest diagnosis from a simple wooden tool. Much of this success is attributable to the remarkable discriminatory powers of the human ear-brain system and to those who made objective studies. It is also likely that this came about because of the wealth of the information contained in this signal.

There are numerous problems. The major problem is that the potential amount of information is too great to be processed readily with currently available instrumentation. Lung sounds have not been modelled adequately. This limits predictions as to the factors which affect them. Their diagnostic utility may also be limited if differing pathological processes produce similar sounds. Indeed, the crackles of congestive heart failure are believed by many to sound similar to those of interstitial fibrosis. Recent observations using advanced analytical techniques suggest that they contain measurable differences. ${ }^{43}$ The extent to which such observations will be fruitful is not yet known. However, precise counting of crackles, their measurement in terms of their individual characteristics, and study of their relative timing in the respiratory cycle is in its infancy. It is likely that noninvasive techniques will allow precise location of the origin of individual crackles within the lung using such techniques as triangulation, developed to detect submarine location. This will provide a means of clearly identifying minute changes in pulmonary pathology within a variable time frame, for example, from minute to minute in intensive care or even from year to year as in monitoring of industrial lung disease. There are many known but quite curious clinical facts that if properly quantified could allow more precision in noninvasive diagnosis. For example, the crackles caused by asbestosis are numerous, while the number of such crackles which occur in sarcoidosis with radiographic changes of equivalent severity are relatively few. Knowing the precise quantity and distribution over the chest wall of such sounds is likely to be useful in diagnosis. It might be argued that such information will require advanced technology which will be too expensive to be practical. This may be true but it is probable that much of the complex instrumentation required to solve the problems outlined above will not be necessary in transposing the advances in knowledge to practical use. An analogy to illustrate this is the demonstration that timing in crackles relates to the presence of bronchitis or obstructive lung disease; early crackles indicate bronchitis, late indicate interstitial fibrosis. ${ }^{40}$ Once the nature of the lung sound is more precisely known, relatively simple means could be developed to study specific aspects which have been shown to be clinically relevant. If the exact variation in frequency content of sound at a specific chest wall site were known in normals, simple devices to detect abnormalities might be developed to screen workers in industrial disease surveys. Electronic processing of sound signals has become greatly simplified with the advances in microcircuitry and the decrease in expense of computer memory. It is unrealistic to think that such devices will not be even more readily available in future years than they currently are.

In summary, if the huge amount of information contained in the lung sounds could be interpreted the potential exists for very powerful diagnostic instruments. To understand lung sounds clearly will require the efforts of a variety of disciplines including anatomy, physiology, pathology, acoustics, mechanical and electrical engineering, psychoacoustics, epidemiology, statistics and computer science. The latter science has tremendous advantages in information processing not possessed by the earbrain system. An interdisciplinary effort has already begun in the form of the International Lung Sounds Association which will have its sixth annual meeting in October. Much of the work presented at the first five meetings has not yet been widely publicised. The problems with interdisciplinary approaches appear to become more complex as each discipline advances. This was not apparently the problem for Laënnec who auscultated patients and performed necropsies. It is almost embarrassing, but indeed a tribute to him, that his work stands now in 1981 as the most comprehensive in correlation of pathology with clinical lung auscultation. His example of careful observation and correlation needs to be followed to turn the adversity of "too much" information to diagnostic advantage.

I would like to thank the following people for thəir 
assistance: Robert G Loudon, Sadamu Ishikawa, Peter Workum, Frank Davidson, Hans Lipp, Stephen Holford, Elizabeth Del Bono, and Lee Starr. The work was supported partly by grants from the Institute of Occupational and Environmental Health, Montreal, PQ, Canada, and by the US National Heart, Lung, and Blood Institute (no HL23318). The table is reproduced with permission from $A$ Simplified Introduction to Lung Sounds by R L H Murphy, published by Stethophonics, PO Box 122, Wellesley Hills, MA02181, USA.

\section{References}

1 Laënnec RTH. A treatise on the diseases of the chest and mediate auscultation. Translated from the French edition by John Forbes. New York: Samuel Wood and Sons, 1935.

2 Bullar JF. Experiments to determine the origin of the respiratory sounds. Proc $R$ Soc London 1884 ; $37: 411$ 23.

3 Hannon RR, Lyman RS. Studies on pulmonary acoustics. II. The transmission of tracheal sounds through freshly exenterated sheep's lungs. Am Rev Turberc 1929; 19:360-75.

4 Martini P, Muller H. Studies on bronchial breathing. Deutsche Arch F Klin Med 1923;143:159-73

5 Forgacs P. Lung sounds. Br J Dis Chest 1969; 63:1-12.

6 Forgacs P, Nath AR, Richardson HD. Breath sounds. Thorax 1971; 26:288-95.

7 Hardin JC, Patterson JL Jr. Monitoring the state of the human airways by analysis of respiratory sound. Acta Astronautica 1979; 6:1137-51.

8 Kraman SS. Determination of the site of production of respiratory sounds by subtraction phonopneumography. Am Rev Respir Dis 1980; 122:303-9.

9 Nairn JR, Turner-Warwick M. Breath sounds in emphysema. Br J Dis Chest 1969; 63:29-37.

10 LeBlanc P, Macklem PT, Ross WRD. Breath sounds and distribution of pulmonary ventilation. $A m$ Rev Respir Dis 1970; 102:10-16.

11 Ploy-Song-Sang Y, Martin RR, Ross WRD, Loudon RG, Macklem P. Breath sounds and regional ventilation. Am Rev Respir Dis 1977; 116:187-99.

12 Rice D. Sound speed in the parenchyma of excised horse lungs. Presented at the Fifth International Lung Sounds Conference, London, England, 1980.

13 Robertson AJ. Rales, rhonchi, and Laënnec. Lancet $1957 ; 1: 417-23$.

14 Schilling RSF, Hughes JPW, Dingwall-Fordyce. Disagreement between observers in an epidemiological study of repiratory disease. $\mathrm{Br} M e d \mathrm{~J} 1955$; 1:65-8.

15 Fletcher CM. The clinical diagnosis of pulmonary emphysema. Proc $R$ Soc Med 1952; 45:577-84.

16 Schneider IC, Anderson AE. Correlation of clinical signs with ventilatory function in obstructive lung disease. Ann Intern Med 1965; 62:477-85.

17 Hudson LD, Conn RD, Matsubara RS, Pribble AH.
Rales-diagnostic uselessness of qualitative adjectives. Am Rev Respir Dis 1978; 113:187.

18 Koran LM. The reliability of clinical methods, data, and judgements. N Engl J Med 1975; 293:642-701.

19 Felson B, Morgan WKC, Bristol LJ, Pendergrass EP, Freesen EL. Observations on the results of multiple readings of chest films in coal miners' pneumoconiosis. Lintonow's Reger RB Radiol 1973; 109:19-23.

20 Cochrane AL. Observer error in the interpretation of chest films. Lancet 1952; 2:505-9.

21 Pardee NE, Martin CJ, Morgan EH. A test of the practical value of estimating breath sound intensity. Chest 1976; 70:341-4.

22 Schneider IC, Anderson AE. Correlation of clinical signs with ventilatory function in obstructive lung disease. Ann Intern Med 1965; 62:477-85.

23 McKusick VA, Jenkins JT, Webb GN. The acoustic basis of the chest examination: studies by means of sound spectrography. Am Rev Tuberc 1955; 72:12-34.

24 Weiss EB, Carlson CJ. Recording of breath sounds. Am Rev Respir Dis 1972; 105:835-9.

25 Grassi C, Marinone G, Morandini C, Pernice A, Puglisi M. Normal and pathological respiratory sounds analyzed by means of a new phonopneumographic apparatus. Respiration 1976; 33:315-24.

26 Cugell DW. Use of tape recordings of respiratory sound and breathing pattern for instruction in pulmonary auscultation. Am Rev Respir Dis 1971; 104:948-50.

27 Wooten FT, Waring WW. Spectral and intensity analysis of respiratory sounds. Proceedings of the 25th annual Conference on Engineering in Medicine and Biology. Arlington, Virginia: Alliance for Engineering in Medicine and Biology, 1972.

28 Ebina T, Toshima M, Fukushi $\mathrm{K}$ et al. Klinische Anwendung elektrisch registrierter Atemgerausche. Beitr Klin Tub 1956; 115:276-81.

29 Klebanov MA. Ronorespirografiia i perspectivy ee ispol'zovaniia. Ter Arkh 1969; 41:57-63.

30 Zizlin DM, Rozenblat VV, Likhacheva EI. Ob'ektivnoe issledovanie dykhatel'nykh shumov s pomoschu'iu chastotnogo analiza. Ter Arkh 1969; 41:108-13.

31 Cabot RC, Dodge HF. Frequency characteristics of heart and lung sounds. JAMA 1925; 84:1793-5.

32 McKusick VA. Cardiovascular sound in health and disease. Baltimore: Williams and Wilkins, 1958.

33 Murphy RLH Jr, Sorensen K. Chest auscultation in the diagnosis of pulmonary asbestosis. J Occup Med 1973; 15:272-6

34 Murphy RLH Jr. Human factors in chest auscultation. In: Picket RM, Triggs TJ, eds. Human factors in health care. Lexington, Massachusetts: DC Heath, 1975.

35 Jalil A, Aronovitch M. Recording and analysis of breath sounds in patients of chest disease. Indian $J$ Chest Dis 1966; 8:158-94.

36 Bass E. Objective recording of sound patterns of normal and pathological breath sounds. Ges Inn Med 1927: 39:345.

37 Forgacs P. Crackles and wheezes. Lancet 1967; 2:203-5. 
38 Forgacs P. Lungsounds. Br J Dis Chest 1969; 63:1-12.

39 Nath AR, Capel LH. Inspiratory crackles and mechanical events of breathing. Thorax 1974; 29:695-8.

40 Nath AR, Capel LH. Inspiratory crackles-early and late. Thorax 1974; 29:223-7.
41 Forgacs P. Lung sounds.. London: Baillière Tindall, 1978.

42 Rubin EH, Rubin M. Thoracic diseases. Philadelphia and London: WB Saunders, 1961.

43 Holford S. Lung sound analysis in the diagnosis of pulmonary asbestosis. SM thesis, MIT, Cambridge, Massachusetts, USA, 1976. 